\title{
Chains on the Eggers tree and polar curves
}

C. T. C. Wall

\begin{abstract}
If $B$ is a branch at $O \in \mathbb{C}^{2}$ of a holomorphic curve, a Puiseux parametrisation $y=\psi(x)$ of $B$ determines 'pro-branches' defined over a sector $|\arg x-\alpha|<\epsilon$. The exponent of contact of two pro-branches is the (fractional) exponent of the first power of $x$ where they differ. We first show how to use exponents of contact to give simple proofs of several well known results.

For $C$ the germ at $O$ of a curve in $\mathbb{C}^{2}$, the Eggers tree $T_{C}$ of $C$ is defined. We also introduce combinatorial invariants (particularly, a certain 1-chain) on $T_{C}$. Any other germ $\Gamma$ at $O$ has contact with $C$ measured by a unique point $X_{\Gamma} \in T_{C}$, and this determines the set of exponents of contact with $C$ of any pro-branch of $\Gamma$. A simple formula establishes the converse, and this leads to a short proof of the theorem on decomposition of a transverse polar of $C$ into parts $P_{i}$, where both the multiplicity of $P_{i}$, and the order of contact with $C$ of each branch $Q$ of $P_{i}$ are explicitly given.
\end{abstract}

\section{Introduction}

We study germs at the origin in $\mathbb{C}^{2}$ of curves $C$ defined by holomorphic equations $f(x, y)=0$. We define a polar curve of $f=0$ to be a curve with equation $P \frac{\partial f}{\partial x}+Q \frac{\partial f}{\partial y}+R f=0$, where $P$ and $Q$ are functions, at least one of which is non-vanishing at the point $O$. This definition is invariant under multiplying the equation either of $f$ or of the polar curve by a function nonvanishing at $O$. One can show that in suitable new coordinates the same curve is given by $g(x, y)=0$ for a suitable $g$, and the same polar curve by $\partial g / \partial y=0$. Each polar curve is associated to a direction at the point $O$.

2000 Mathematics Subject Classification: $14 \mathrm{H} 20$.

Keywords: Eggers tree, exponent of contact, polar curve, decomposition. 
In the general case this is $Q(O) x=P(O) y$; and for $\partial f / \partial y=0$ it is $x=0$. The polar is called a transverse polar if this line is transverse to $C$ at $O$.

It is natural to ask to what extent the type of singularity of $C$ at $O$ determines that of the polar curve $P$. The multiplicity of $P$ is 1 less than that of $C$ if and only if we have a transverse polar, so from now on we will restrict to this case.

It has long been known that the germ $P$ is usually reducible. In 1976 a general statement was obtained by Michel Merle [14], under the assumption that $C$ consists of a single branch: this result yields a (local) decomposition $P=\bigcup_{1 \leq i \leq g} P_{i}$, where both the multiplicity of $P_{i}$, and the order of contact with $C$ of each branch $Q$ of $P_{i}$ are given. However, examples show that the number of branches can vary for different transverse polars of the same curve, and that $P_{i}$ need not even be reduced.

Merle's result raised the problem of finding a corresponding statement for polars of curves with several branches. A general theorem is now known (see e.g. [10]), but all known proofs involve messy combinatorial arguments. In this paper we reduce these to easy calculations by introducing additional structure on the Eggers tree.

The main tool used in our argument is the notion of exponent of contact. We begin by presenting this notion, and using it to give simple proofs of several well known results. This gives an introduction to our tools as well as a basis for the arguments to follow. We refer to [2] and [7] for background information in this area.

\section{Preliminaries}

Suppose the curve $C$ given near $O$ by a holomorphic equation $f(x, y)=0$, and that the $y$-axis is transverse to $C$ at $O$. There is a factorisation (unique up to units) $f=\prod f_{j}$ with each $f_{j}$ irreducible; correspondingly, $C$ is a union of irreducible curve-germs $B_{j}$, its branches. We assume $C$ reduced, so these are mutually distinct.

For a single branch $B$, with equation $f=0$, the Weierstrass preparation theorem tells us that we may adjust $f$ by a unit to be a monic polynomial in $y$. The degree $m$ of this polynomial is equal to the multiplicity $m(B)$ of $B$. By Puiseux' theorem, we may solve the equation $f=0$ to write $y$ as a fractional power series in $x$

$$
y=\sum_{r \geq m} a_{r} x_{m}^{r}, \quad \text { with } x_{m}^{m}=x .
$$

The $m$ roots of the equation are obtained by taking successively for $x_{m}$ the $m^{\text {th }}$ roots of $x$. 
We want to distinguish a single such root. To do this it is necessary to restrict $x$ to lie in a sector $|\arg x-\alpha|<\epsilon$ of the plane of complex numbers. Fixing a root gives what we will call a pro-branch defined over this sector. If the sector contains the positive real axis, we can choose $x_{m}$, for any $m$, to take positive real values on the positive real axis, so we can write fractional powers of $x$ without ambiguity. Although the terminology 'pro-branch' is new, making a choice of $x_{m}$ (in fixed coordinates) is standard procedure.

Suppose $\gamma, \gamma^{\prime}$ are pro-branches of branches $B, B^{\prime}$, defined over the same sector. We may write their equations in the form $y=\sum_{s} c_{s} x^{s}, y=\sum_{s} c_{s}^{\prime} x^{s}$, where the exponents $s$ satisfy $s \geq 1$ and $m(B) s \in \mathbb{Z}, m\left(B^{\prime}\right) s \in \mathbb{Z}$ respectively. We define the exponent of contact to be $\mathcal{O}\left(\gamma, \gamma^{\prime}\right):=\min \left\{s \mid c_{s} \neq c_{s}^{\prime}\right\}$. This may also be expressed geometrically:

$$
\mathcal{O}\left(\gamma, \gamma^{\prime}\right)=\lim _{P \in \gamma, P \rightarrow O} \frac{\log \left(\operatorname{dist}\left(P, \gamma^{\prime}\right)\right)}{\log (\operatorname{dist}(P, O))}
$$

so is independent of coordinates. It will be convenient to make the convention that $\mathcal{O}(\gamma, \gamma)=\infty$. If we have 3 pro-branches $\gamma, \gamma^{\prime}$ and $\gamma^{\prime \prime}$ then in all cases

$$
\mathcal{O}\left(\gamma^{\prime \prime}, \gamma\right) \geq \min \left(\mathcal{O}\left(\gamma, \gamma^{\prime}\right), \mathcal{O}\left(\gamma^{\prime}, \gamma^{\prime \prime}\right)\right)
$$

so the two smaller of the three orders coincide.

Lemma 1.1 The set of $m(B)$ numbers (counted with multiplicities) given by the orders of contact of a pro-branch $\gamma^{\prime}$ of $B^{\prime}$ with all the pro-branches of $B$ over the same sector is independent of the choices of sector and of $\gamma^{\prime}$.

Proof: The exponents of contact are not affected if we shrink the sector. Each exponent of contact is also not changed if we rotate the sector round the origin in $\mathbb{C}$, using analytic continuation; hence the set as a whole is unaltered. But a full rotation round $O$ permutes the pro-branches of $B^{\prime}$ transitively, so the set is also independent of the choice of $\gamma^{\prime}$.

This result allows us to use pro-branches without reference to choices. We may fix a sector, and write $\operatorname{pro}(B)$ for the set of all pro-branches of $B$ (over that sector), so the set defined above may be written $\left\{\mathcal{O}\left(\gamma^{\prime}, \gamma\right) \mid \gamma \in \operatorname{pro}(B)\right\}$. We emphasise that although the set is unordered, we do count its elements with multiplicities.

The exponent of contact of the branches is defined as

$$
\mathcal{O}\left(B, B^{\prime}\right)=\min \left\{\mathcal{O}\left(\gamma, \gamma^{\prime}\right) \mid \gamma \in \operatorname{pro}(B), \gamma^{\prime} \in \operatorname{pro}\left(B^{\prime}\right)\right\}
$$

Here we may fix $\gamma$ or $\gamma^{\prime}$ (but not, of course, both). It follows again that given three branches $B, B^{\prime}$ and $B^{\prime \prime}$, the two smaller of the three numbers $\mathcal{O}\left(B, B^{\prime}\right), \mathcal{O}\left(B, B^{\prime \prime}\right)$ and $\mathcal{O}\left(B^{\prime}, B^{\prime \prime}\right)$ coincide. We have $\mathcal{O}\left(B, B^{\prime}\right)=\infty$ if and only if $B=B^{\prime}$. 
Suppose the branch $B$ with $m(B)=m$ given as in (1.1). The Puiseux characteristic is defined inductively by $e_{0}:=m$ and

$$
\beta_{q}:=\min \left\{r \mid a_{r} \neq 0, a_{r} \text { NOT divisible by } e_{q-1}\right\}, \quad e_{q}:=\operatorname{hcf}\left(e_{q-1}, \beta_{q}\right),
$$

the procedure terminating when we arrive (as we must) at $e_{g}=1$ for some $g$. It is well known that the Puiseux characteristic $\left(m ; \beta_{1}, \ldots, \beta_{g}\right)$ determines the topological type of $B$, and conversely. It will be convenient to write $\beta_{g+1}:=\infty$. To simplify our formulae, we also write $\alpha_{q}:=\beta_{q} / m$ for each $q$.

Proposition 1.2 Let $\gamma^{\prime} \in \operatorname{pro}\left(B^{\prime}\right)$, let $\mathcal{O}\left(B, B^{\prime}\right)=\kappa$, and let $\alpha_{q}<\kappa \leq$ $\alpha_{q+1}$. Then

(i) $\left\{\mathcal{O}\left(\gamma^{\prime}, \gamma\right): \gamma \in \operatorname{pro}(B)\right\}$ consists of:

$$
\begin{aligned}
& \alpha_{i} \text {, occurring }\left(e_{i-1}-e_{i}\right) \text { times }(1 \leq i \leq q) \text {; } \\
& \kappa \text {, occurring } e_{q} \text { times. }
\end{aligned}
$$

(ii) Let $\gamma \in \operatorname{pro}(B)$. Then $\left\{\mathcal{O}\left(\gamma, \gamma^{\prime}\right): \gamma^{\prime} \in \operatorname{pro}(B), \gamma^{\prime} \neq \gamma\right\}$ consists of: $\alpha_{i}$, occurring $\left(e_{i-1}-e_{i}\right)$ times $(1 \leq i \leq g)$.

(iii) The intersection number B.B' $B^{\prime}$ is equal to the sum

$$
\sum_{\gamma \in \operatorname{pro}(B), \gamma^{\prime} \in \operatorname{pro}\left(B^{\prime}\right)} \mathcal{O}\left(\gamma, \gamma^{\prime}\right)
$$

(iv) Let $\mathcal{O}\left(B, B^{\prime}\right)=\kappa$ and $\alpha_{q}<\kappa \leq \alpha_{q+1}$. Then

$$
B . B^{\prime}=\frac{m\left(B^{\prime}\right)}{m(B)}\left\{\left(e_{0}-e_{1}\right) \beta_{1}+\ldots+\left(e_{q-1}-e_{q}\right) \beta_{q}+e_{q} m(B) \kappa\right\} .
$$

Proof: (i) Suppose the pro-branch $\gamma^{\prime}$ has exponent of contact $\kappa$ with the pro-branch $\gamma$ given by (1.1). The remaining pro-branches $\gamma_{s}$ of $B$ in the chosen sector are obtained by multiplying $x_{m}$ formally by $e^{2 \pi i s / m}$ for $0<s<m$. The result now follows by comparing the coefficients in the resulting series.

In view of our conventions, assertion (ii) is a special case of (i).

(iii) Intersection numbers may be calculated by choosing a (reduced) equation $f(x, y)=0$ for $B^{\prime}$, a parametrisation $(x, y)=(a(t), b(t))$ for $B$, substituting, and taking the order of the result $f(a(t), b(t))$ as a function of $t$.

Set $f=\prod_{k=1}^{m\left(B^{\prime}\right)}\left(y-b_{k}^{\prime}(x)\right)$, where the $b_{k}^{\prime}$ are Puiseux series, and take the parametrisation as $\left(t^{m(B)}, b(t)\right)$. We thus obtain $\sum_{k=1}^{m\left(B^{\prime}\right)} \operatorname{ord}_{t}\left(b(t)-b_{k}^{\prime}\left(t^{m(B)}\right)\right)$. 
Since $x=t^{m(B)}$, ord $t=m(B) \operatorname{ord}_{x}$. The order in $x$ of the difference is precisely the exponent of contact of the pro-branches. We thus obtain, for a chosen pro-branch $\gamma$ of $B, m(B) \sum_{\gamma^{\prime} \in \operatorname{pro}\left(B^{\prime}\right)} \mathcal{O}\left(\gamma, \gamma^{\prime}\right)$.

(iv) is now immediate.

That, given the numerical data for the branch $B$, and $m\left(B^{\prime}\right)$, the exponent of contact $\mathcal{O}\left(B, B^{\prime}\right)$ and the intersection number $B . B^{\prime}$ determine each other, is not of course new; it goes back to M. Noether.

\section{The Eggers tree}

We next describe a convenient notation for orders of contact of a curve with several branches. This was first given in in the thesis [9] of Harald Eggers. We associate with it combinatorial information going beyond that of Eggers.

Begin with a single branch $B$. We define the tree $T_{B}$ to be a copy of the compactified positive real axis, which we denote $[0, \infty]$, and write

$$
v_{B}: T_{B} \rightarrow[0, \infty], \quad \pi_{B}:[0, \infty] \rightarrow T_{B}
$$

for inverse isomorphisms. The vertices of $T_{B}$ are $A_{0}:=\pi_{B}(0), A_{q}:=$ $\pi_{B}\left(\alpha_{q}\right)$ for $1 \leq q \leq g$, and $B^{\infty}:=\pi_{B}(\infty)$. Write $\nu_{B}$ for the function defined on 1-simplices by $\nu_{B}\left[A_{q-1} A_{q}\right]:=m /\left(e_{q-1}\right)$, so in particular we have $\nu_{B}\left[A_{0} A_{1}\right]=m / e_{0}=1$ and $\nu_{B}\left[A_{g} B^{\infty}\right]=m / e_{g}=m$. We also write $\nu_{B}$ for the 1-chain on the simplicial complex $T_{B}$ with these coefficients.

Now suppose we have two branches $B, B^{\prime}$; use the notation of Proposition 1.2. We define $T_{B \cup B^{\prime}}$ as obtained from $T_{B} \cup T_{B^{\prime}}$ by identifying the images of $[0, \kappa]$ under $\pi_{B}$ and $\pi_{B^{\prime}}$. Let us check that the identification is compatible with the structures we have defined. Since the exponent of contact is $\kappa>\alpha_{q-1}$, there are Puiseux series of $B$ and $B^{\prime}$ which agree up to (but not including) the term in $x^{\kappa}$. It follows from the definition of the Puiseux characteristic that, for all $i<q, \alpha_{i}=\beta_{i} / m=\beta_{i}^{\prime} / m^{\prime}$, and $e_{i} / m=e_{i}^{\prime} / m^{\prime}$. Hence the points $A_{i}$ on the common interval [0, $\kappa$ [match up, as do the values of $\nu$ for the common edges (including $\pi_{B}\left[\alpha_{q-1}, \kappa\right]$ ). We introduce the branch point $\pi_{B}(\kappa)$ as a vertex even if it coincides with neither of the points $A_{q}$.

In general, let the branches of $C$ be $\left\{B_{j} \mid j \in J\right\}$, with $\kappa_{j, k}:=\mathcal{O}\left(B_{j}, B_{k}\right)$, and define $T_{C}$ to be the quotient of the disjoint union of the $T_{B_{j}}$ by the identification, for any pair $j, k$, of the images of $\left[0, \kappa_{j, k}\right]$ under $\pi_{B_{j}}$ and $\pi_{B_{k}}$. More precisely, define a relation on $[0, \infty] \times J$ by $(a, j) \sim(a, k)$ if $a \leq \kappa_{j, k}$. This is clearly reflexive and symmetric; it is transitive since if $(a, j) \sim(a, k)$ and $(a, k) \sim(a, l)$ then $a \leq \min \left(\kappa_{j, k}, \kappa_{k, l}\right) \leq \kappa_{j, l}$, by $(1.2)$, so $(a, j) \sim(a, l)$. 
We define $T_{C}$ to be the quotient of $[0, \infty] \times J$ by this equivalence relation: this is a tree. The inclusion of $[0, \infty] \times j$ induces an injection $\pi_{B_{j}}:[0, \infty] \rightarrow$ $T_{C}$, whose image we may identify with $T_{B_{j}}$. The first projection induces a map $v: T_{C} \rightarrow[0, \infty]$, whose restriction to $T_{B_{j}}$ is $v_{B_{j}}$.

We regard $T_{C}$ as a graph whose vertices are the images of those defined for the separate branches, together with such branch points $I_{j, k}:=\pi_{B_{j}}\left(\kappa_{j, k}\right)$ as are not already included. The values of $\nu$ on edges (subdivided, as necessary, by points $I_{j, k}$ ) agree as before, so the 1-chains $\nu_{B_{j}}$ fit together to give a single 1-chain $\nu$ on the 1-complex $T_{C}$. We orient each edge of $T_{C}$ in the sense in which $v$ increases: thus all edges point away from the vertex $A_{0}$. We may regard the set of vertices of $T_{C}$ as a poset with this ordering. Then $I_{j, k}=$ $\inf \left(B_{j}^{\infty}, B_{k}^{\infty}\right)$. We can identify $T_{C}$ with the Hasse diagram of the poset.

Lemma 2.1 For any branch $\Gamma$ at $O$ there is a unique point $X_{\Gamma} \in T_{C}$ such that for each $j \in J, \mathcal{O}\left(\Gamma, B_{j}\right)=v\left(\inf \left(X_{\Gamma}, B_{j}^{\infty}\right)\right)$.

Proof: Choose $j$ to maximise $\mathcal{O}\left(\Gamma, B_{j}\right)$-the value is $\mathcal{O}(\Gamma, C)=\kappa$, say - and define $X_{\Gamma}:=\pi_{B_{j}}(\kappa)$. Then by the triangle inequality for orders of contact, for any $k \neq j$ we either have $\mathcal{O}\left(\Gamma, B_{k}\right)=\kappa \leq \mathcal{O}\left(B_{j}, B_{k}\right)$ or $\mathcal{O}\left(\Gamma, B_{k}\right)=\mathcal{O}\left(B_{j}, B_{k}\right) \leq \kappa$. In the former case, $X_{\Gamma} \leq I_{j, k}<B_{k}^{\infty}$, so $v\left(\inf \left(X_{\Gamma}, B_{j}^{\infty}\right)\right)=v\left(X_{\Gamma}\right)=\kappa=\mathcal{O}\left(\Gamma, B_{k}\right)$. In the latter case, $X_{\Gamma} \cap B_{k}^{\infty}=$ $I_{j, k}$, so $v\left(\inf \left(X_{\Gamma}, B_{j}^{\infty}\right)\right)=v\left(I_{j, k}\right)=\mathcal{O}\left(B_{j}, B_{k}\right)=\mathcal{O}\left(\Gamma, B_{k}\right)$. Uniqueness is immediate.

We measure the contact of $\Gamma$ with $C$ by the 0 -chain $[\Gamma]:=m(\Gamma) X_{\Gamma}$. (The point $X_{\Gamma}$ need not be a vertex of $T_{C}$ as we have defined it, so in any given situation a finite amount of subdivision may be necessary.) In particular, $\left[B_{j}\right]=m\left(B_{j}\right) B_{j}^{\infty}$. For a curve germ $\Delta$ with several branches $\Gamma_{i}$, the contact with $C$ is measured by the 0 -chain $[\Delta]:=\sum_{i}\left[\Gamma_{i}\right]$. Thus $[C]=$ $\sum_{j \in J} m\left(B_{j}\right) B_{j}^{\infty}$.

Since $T_{C}$ is a tree, its chain groups (with $\mathbb{Z}$ coefficients) form a short exact sequence $0 \rightarrow C_{1} \stackrel{\partial}{\longrightarrow} C_{0} \stackrel{\epsilon}{\longrightarrow} \mathbb{Z} \rightarrow 0$, which is split by the map from $\mathbb{Z}$ to $C_{0}$ taking 1 to $A_{0}$. This induces a splitting map $s: C_{0} \rightarrow C_{1}$. The value of $s$ on a vertex $X$ is the sum of the edges forming the path from $A_{0}$ to $X$.

For any vertex $X$, write $\widehat{X}$ for the 1-cochain dual to the edge immediately below $X$ (and $\widehat{A_{0}}:=0$ ). Now if $\Delta$ is, as above, a germ with branches $\Gamma_{i}$, we claim that $\langle\widehat{X}, s[\Delta]\rangle$ is the sum of the multiplicities of the branches $\Gamma$ of $\Delta$ passing through $X$ (i.e. with $X \leq X_{\Gamma}$ ). It suffices to check for a single branch $\Gamma$. But then $s[\Gamma]$ is the sum of the edges below $X_{\Gamma}$, each with coefficient $m(\Gamma)$, so has non-trivial product with $\widehat{X}$ if and only if $X \leq X_{\Gamma}$. 
Let us calculate the list of orders of contact with $C$ of the pro-branches of a general branch $\Gamma$. First consider the case when $C$ has a single branch $B$.

By (1) of Proposition 1.2, if $\gamma^{\prime}$ is a pro-branch of $\Gamma, \mathcal{O}(B, \Gamma)=\kappa$, and $\alpha_{q}<\kappa \leq \alpha_{q+1}$, then $\left\{\mathcal{O}\left(\gamma^{\prime}, \gamma\right) \mid \gamma \in \operatorname{pro}(B)\right\}$ consists of $\alpha_{i}$, occurring $\left(e_{i-1}-e_{i}\right)$ times $(1 \leq i \leq q)$; and $\kappa$, occurring $e_{q}$ times. We may represent this list by the 0 -chain $\sum_{1}^{q}\left(e_{i-1}-e_{i}\right) A_{i}+e_{q} X_{\Gamma}$ on $T_{B}$. Applying the splitting map $s$ to this 0 -chain gives the 1-chain

$$
\zeta_{B, \Gamma}:=\sum_{1}^{q} e_{r-1}\left(A_{r-1} A_{r}\right)+e_{r}\left(A_{q} X_{\Gamma}\right)
$$

and the 0-chain is then $\partial \zeta_{B, \Gamma}+m(B) A_{0}$. Observe that $\left\langle\widehat{M}, \zeta_{B, \Gamma}\right\rangle$ vanishes unless $M \leq X_{\Gamma}$, and is then $e_{r-1}$ if $A_{r-1}<M \leq A_{r}$.

If $C$ has branches $B_{j}$, we define $\zeta_{C, \Gamma}:=\sum_{j} \zeta_{B_{j}, \Gamma}$. This is determined by the list of orders of contact of a pro-branch of $\Gamma$ with the pro-branches of the several branches of $C$, so depends only on the point $X_{\Gamma}$. We define $\eta_{C}\left(X_{\Gamma}\right):=\zeta_{C, \Gamma}$, and extend to an additive homomorphism $\eta_{C}: C_{0} \rightarrow C_{1}$. Thus if $\Delta$ has branches $\Gamma_{i}$, we have $\eta_{C}[\Delta]:=\sum_{i} m\left(\Gamma_{i}\right) \zeta_{C, \Gamma_{i}}$.

For each point $M \neq A_{0}$, the highest point occurring with non-zero coefficient in $\partial \eta_{C}(M)$ is $M$ itself. Thus the matrix of $\eta_{C}$ is triangular, and $\eta_{C}$ has kernel $\mathbb{Z} A_{0}$. Hence to recover the characteristic 0 -cycle $[\Delta]$ of the curve-germ $\Delta$ it is sufficient to know the information $\eta_{C}[\Delta]$ determined by the orders of contact. This remark applies not only to the tree $T_{C}$ but also to any tree obtained by subdividing at a finite number of points: the transitions $\eta_{C}$ and its inverse do not require additional subdivision.

The explicit inversion of $\eta_{C}$ is given by the following.

Lemma 2.2 With the above notation, we have

$$
\langle\widehat{M}, \nu\rangle\left\langle\widehat{M}, \eta_{C}[\Delta]\right\rangle=\langle\widehat{M}, s[\Delta]\rangle\langle\widehat{M}, s[C]\rangle
$$

Proof: Since both sides are additive in $[\Delta]$, we may assume $\Delta=\Gamma$ irreducible. Now calculate explicitly. For any point $M$, we have

$$
\left\langle\widehat{M}, \eta_{C}[\Gamma]\right\rangle=\sum_{j} m(\Gamma)\left\langle\widehat{M}, \zeta_{B_{j}, \Gamma}\right\rangle=\sum_{j} m(\Gamma) m\left(B_{j}\right) c_{j}(M)
$$

where $c_{j}(M)$ is zero unless $M \leq X_{\Gamma}$ and $M \in T_{B_{j}}$, while if $M \leq X_{\Gamma}$ and $\left.\left.M \in \pi_{B_{j}}\right] A_{r-1}, A_{r}\right]$, we have $c_{j}(M)=e_{r-1} / m\left(B_{j}\right)=1 /\langle\widehat{M}, \nu\rangle$-though the definition of $e_{r-1}$ depends on the choice of branch, the quotient $e_{r-1} / m$ is determined by the point of $T_{C}$. 
Hence the right hand side of $(2.2)$ is 0 unless $M \leq X_{\Gamma}$, when it becomes

$$
m(\Gamma) \sum_{M \leq B_{j}^{\infty}} m\left(B_{j}\right) /\langle\widehat{M}, \nu\rangle=m(\Gamma)\langle\widehat{M}, s[C]\rangle /\langle\widehat{M}, \nu\rangle .
$$

Thus in all cases it is equal to $\langle\widehat{M}, s[\Gamma]\rangle\langle\widehat{M}, s[C]\rangle /\langle\widehat{M}, \nu\rangle$.

\section{Decomposition of polar curves}

Merle's decomposition theorem was extended to the case of curves $C$ with two branches by Delgado [8], who also showed that the method of proof did not work for curves with more branches. A number of detailed results on singularities of $P$ were obtained by Casas [3], [4], [5], [6], particularly for the case when $C$ is a general member of its equisingularity class, but simple examples show that the equisingularity class of $P$ is not determined by that of $C$. A result on decomposition in general was obtained by Lê, Michel and Weber [13]. A full generalisation of Merle's theorem was obtained in the 1996 thesis of García Barroso [10], [11]. Another proof was found by Assi [1]. However the problem was essentially already solved in 1983 by Eggers [9].

We give a short proof which, like those cited, hinges on a result of Kuo and $\mathrm{Lu}[12]$, which we may state as follows.

Proposition 3.1 [12, Lemma 3.3] Let $C$ be a reduced curve germ, $P$ a transverse polar of $C$. Then for any pro-branch $\gamma$ of $C$,

$$
\{\mathcal{O}(\gamma, \delta) \mid \delta \in \operatorname{pro}(P)\}=\left\{\mathcal{O}\left(\gamma, \gamma^{\prime}\right) \mid \gamma \neq \gamma^{\prime} \in \operatorname{pro}(C)\right\}
$$

The proof depends on the use of Newton polygons.

Theorem 3.2 Let $C$ be a reduced curve germ, $P$ a transverse polar curve of $C$. Then the contact of $P$ with $C$ is measured by the 0-chain $[P]=$ $[C]-\partial \nu-A_{0}$. In particular, the points occurring with non-zero coefficients in $[P]$ are the vertices of $T_{C}$ with values in $] 0, \infty[$.

Proof: By Lemma 3.1, for any pro-branch $\gamma$ of $C$,

$$
\{\mathcal{O}(\gamma, \delta) \mid \delta \in \operatorname{pro}(P)\}=\left\{\mathcal{O}\left(\gamma, \gamma^{\prime}\right) \mid \gamma \neq \gamma^{\prime} \in \operatorname{pro}(C)\right\}
$$

Thus if $\gamma \in \operatorname{pro}\left(B_{j}\right)$, the 0 -chains corresponding to $\{\mathcal{O}(\gamma, \delta) \mid \delta \in \operatorname{pro}(\Gamma)\}$ for $\Gamma$ equal to $P$ or $C$ differ by $B_{j}^{\infty}$. Hence the 1 -chains differ by $s\left(B_{j}^{\infty}\right)$, and

$$
\eta_{C}[P]=\sum_{j} m\left(B_{j}\right)\left(\eta_{C}\left[B_{j}^{\infty}\right]-s\left[B_{j}^{\infty}\right]\right)=\eta_{C}[C]-s[C]
$$


Now applying (2.1) to $\Gamma=P$, for any point $M$ of the tree,

$$
\langle\widehat{M}, s[P]\rangle\langle\widehat{M}, s[C]\rangle=\langle\widehat{M}, \nu\rangle\left\langle\widehat{M}, \eta_{C}[P]\right\rangle=\langle\widehat{M}, \nu\rangle\left\langle\widehat{M},\left(\eta_{C}[C]-s[C]\right)\right\rangle,
$$

while, applying (2.1) to $\Gamma=C$ itself, we have

$$
\langle\widehat{M}, s[C]\rangle\langle\widehat{M}, s[C]\rangle=\langle\widehat{M}, \nu\rangle\left\langle\widehat{M}, \eta_{C}[C]\right\rangle
$$

Substituting (3.3) in the right hand side of (3.2), and cancelling the factor $\langle\widehat{M}, s[C]\rangle$, now gives $\langle\widehat{M}, s[P]\rangle=\langle\widehat{M}, s[C]\rangle-\langle\widehat{M}, \nu\rangle$, and since the 1-cochains $\widehat{M}$ span $C^{1}$ we infer $s[P]=s[C]-\nu$.

Applying $\partial$ we see that $[P]$ differs from $[C]-\partial \nu$ by a multiple of $A_{0}$. We determine this multiple by applying $\epsilon: \epsilon[P]=m(P)=m(C)-1, \epsilon[C]=$ $m(C)$, and $\epsilon \circ \partial=0$, so $[P]=[C]-\partial \nu-A_{0}$, as claimed.

By inspection, the coefficients of $A_{0}$ and the $B_{j}^{\infty}$ in this expression vanish (as they must), and no point other than the original vertices of $T_{C}$ can appear. To see that each of these has non-zero coefficient, observe that for a single branch, the values of $\nu$ on the edges increase strictly at each $A_{i}$, so each $A_{i}$ occurs with strictly positive coefficient in $-\partial \nu$; while for a branch point $I_{j, k}$ the values of $\nu$ on each of the edges immediately above the point are at least equal to the value on the edge immediately below, so again we have a strictly positive coefficient.

\section{References}

[1] Assi, A.: Partial derivatives of a meromorphic plane curve. Preprint, Angers, 1998.

[2] Brieskorn, E. AND KnÖrrer, H.: Plane Algebraic Curves. Birkhäuser, 1986.

[3] Casas-Alvero, E.: On the singularities of polar curves. Manuscripta Math. 43 (1983), 167-190.

[4] Casas-Alvero, E.: Infinitely near imposed singularities and singularities of polar curves. Math. Ann. 287 (1990), 429-494.

[5] Casas-Alvero, E.: Base points of polar curves. Ann. Inst. Fourier $4 \mathbf{1}$ (1991), 1-10.

[6] Casas-Alvero, E.: Singularities of polar curves. Compositio Math. 89 (1993), 339-359.

[7] Casas-Alvero, E.: Singularities of plane curves. Cambridge University Press, 1999.

[8] Delgado de la Mata, F.: A factorisation theorem for the polar of a curve with two branches. Comp. Math. 92 (1994), 327-375. 
[9] Eggers, H.: Polarinvarianten und die Topologie von Kurvensingularitäten. Bonner Math. Scrift 147, 1982.

[10] García Barroso, E.: Invariants des singularités de courbes planes et courbure des fibres de Milnor. Doctoral Thesis, La Laguna, 1996.

[11] García Barroso, E.: Sur les courbes polaires d'une courbe plane réduite. Proc. London Math. Soc. 81 (2000), 1-28.

[12] KuO, T.-C. And Lu, Y.C.: On analytic function-germs of two complex variables. Topology 16 (1977), 299-310.

[13] Lê, D.-T., Michel, F. And Weber, C.: Courbes polaires et topologie des courbes planes. Ann. Sci. École Norm. Sup. 24 (1991), 141-169.

[14] Merle, M.: Invariants polaires de courbes planes. Invent. Math. 41 (1977), 103-111.

Recibido: 22 de marzo de 2002

Revisado: 21 de octubre de 2002

C. T. C. Wall

Department of Mathematical Sciences

University of Liverpool

Liverpool L69 7ZL, England

ctcw@liv.ac.uk 